\title{
The Multi-Station Based Variable Speed Limit Model for Realization on Urban Highway
}

\author{
Soobin Jeon ${ }^{1} \mathbb{D}$, Chongmyung Park ${ }^{2}$ and Dongmahn Seo ${ }^{1, * \mathbb{C}}$ \\ 1 Department of Computer Engineering, School of Software Convergence, Catholic University of Daegu, \\ Gyeongsan-si 712010, Korea; marsberry@cu.ac.kr \\ 2 Department of Computer and Communications Engineering, School of Information Technology, \\ Kangwon National University, Chuncheon-si 200701, Korea; chongmyung.park@gmail.com \\ * Correspondence: sarum@cu.ac.kr; Tel.: +82-010-4053-5447
}

Received: 24 March 2020; Accepted: 6 May 2020; Published: 13 May 2020

\begin{abstract}
Intelligent transport systems (ITS) are a convergence of information technology and transportation systems as seen in the variable speed limit (VSL) system. Since the VSL system controls the speed limit according to the traffic conditions, it can improve the safety and efficiency of a transport network. Many researchers have studied the real-time VSL (RVSL) algorithm based on real-time traffic information from multiple stations recording traffic data. However, this method can suffer from inaccurate selection of the VSL start station (VSS), incorrect VSL calculations, and is unable to quickly react to the changing traffic conditions. Unstable VSL systems result in more congestion on freeways. In this study, an enhanced VSL algorithm (EVSL) is proposed to address the limitations of the existing RVSL algorithm. This selects preliminary VSL start stations (pVSS), which is expected to end congestion using acceleration and allocates final VSSs for each congestion interval using selected pVSS. This controls the vehicles that entered the congestion area based on the selected VSS. We used four metrics to evaluate the performance of the proposed VSL (VSS stability assessment, speed control stability assessment, travel time, and shockwave), which were all enhanced when compared to the standard RVSL algorithm. In addition, the EVSL algorithm showed stable VSL performance, which is critical for road safety.
\end{abstract}

Keywords: variable speed limit; traffic control; travel time; intelligent transportation system

\section{Introduction}

Intelligent transportation systems (ITS) are integrated traffic systems based on the convergence of civil engineering and information technology fields. Such systems aim to provide a low cost/high efficiency traffic management system that enhances traffic safety. Several studies have been performed with the aim of reducing traffic congestion and alleviating the risk of car accidents on urban expressways. Both variable speed limit (VSL) and ramp metering technologies are representative methods for improving traffic conditions on expressways [1]. When vehicle accidents and traffic shockwaves are present, sudden variations in traffic flow are inevitable, which increase the risk of further accidents. In the VSL system, speed indicators are installed on the roads at regular intervals and the system collects traffic information in real time. Based on this information, the VSL system controls traffic flow by applying suitable speed limits in the regions suffering from traffic congestion. Hence, the VSL system can alleviate traffic congestion. In addition, a major advantage is its fast response to sudden events, such as road repair works and vehicle accidents. Since the VSL system enhances road safety, it is an important solution for upcoming ITS [2,3].

Existing VSL systems [4] used traffic information collected from single stations, which are devices to detect vehicles as a like magnetic loop detector, deployed in the different sections of road. 
This approach has already contributed to improving traffic congestion. However, in the case of urban expressway environments where the vehicles are travelling at high speed, an appropriate VSL system should consider the traffic conditions detected by multiple stations since traffic in one area can affect other parts of the expressway. Hence, recent VSL studies proposed the real-time variable speed limit (RVSL) method [5-8], which focuses on traffic control between multiple stations.

Especially, Jo et al. (JVSL) [7] is one of the most recently studied RVSL models and shows a very good performance from the freeway in Twin City, Minnesota. Although this algorithm shows good performance, it could not be applied to the highway due to some problems as below. It is operated next. First, it was found that the congestion area of the freeway contained multiple VSL sections. In addition, it defines the VSL start station (VSS) and controls the speed limit of vehicles in real time. However, often, the JVSL cannot determine the correct VSS. Errors occur in the calculation of the VSL for each station, which can shut down the entire system. This often happens with legacy RVSL systems. Since the purpose of VSL is to improve the safety on roads, if the VSL system cannot stably control traffic flow, traffic congestion will not be alleviated and could be aggravated over time.

Based on the VSS, the VSL algorithm controls the speed of the vehicles entering the congested section of the road. To control the speed, the VSL displays the modified speed value on a dynamic message sign (DMS), which is visible to drivers and located at a different location than the station. Thus, the VSL system must control the speed of vehicles based on the location of the DMS. However, the JVSL model calculates the VSL value of each station based on the position of the VSS instead of the location of the DMS. Since the stations and DMS are in different areas, an accurate VSL value cannot be displayed on each DMS device.

In this study, an enhanced VSL algorithm (EVSL) is proposed to address limitations of the existing JVSL algorithm. The proposed algorithm enhances the safety on the freeway because it provides a stable VSL for multiple stations. The proposed algorithm is composed of three parts. First, the moving average speed is calculated to achieve the stable speed data of each station. Second, a new method is proposed for making VSS assignments to accurately determine the VSS. Third, a new method calculates the VSL value of each station based on the VSS and DMS locations, instead of using only the station location. Lastly, the new VSL is applied to the DMS of each station. To evaluate the proposed algorithm, we constructed a simulation environment presenting real road conditions (outer ring road in the south of Seoul, South Korea). The PTV VISSIM software was used to simulate our proposed algorithm. Calibration of the system using the raw data was performed to make it suitable for use in a similar real traffic environment. The proposed algorithm was compared to the existing JVSL algorithm considering the efficiency and stability. The proposed VSL method showed better performance for all four metrics used to evaluate the performance (VSS stability assessment, speed control stability assessment, travel time, and shockwave).

The remainder of this paper is organized as follows. Section 2 reviews Existing JVSL algorithms and discusses the problem of them. Section 3 proposes an enhanced VSL algorithm to solve JVSL problems. Section 4 describes the experimental environment to simulate a real traffic environment. Section 5 offers measurement and evaluation of the performance of the proposed enhanced VSL algorithm. Section 6 concludes the paper.

\section{Control Design and Problems of JVSL}

The VSL system is being employed to reduce traffic accidents on urban freeways. Traffic accidents and shockwaves on the road suddenly affect the speed of vehicles, which can cause additional accidents [9]. To solve this problem, the VSL system collects real-time traffic information, which is used to determine an appropriate speed limit for each section of the freeway suffering traffic congestion. With an active VSL system in place, the vehicles reduce their speed before entering the congested section, which reduces the risk of accidents and improves road safety. The VSL system is composed of three elements including sensors monitoring the traffic conditions, the control system processing the sensing data, and the DMS devices displaying the VSL. Since the VSL enhances the stability of 
traffic flow and reduces driver stress, it is widely applicable. Specifically, to improve the stability of freeway conditions, several studies measured traffic data and analyzed them in real time. These studies focused on data such as the speed, density, and volume of traffic. In addition, some studies proposed an integrated system including both VSL and ramp metering on interconnections [10,11].

Previous studies developed VSL algorithms based on information received about the road conditions, which contributed somewhat to road safety. However, since these studies were based on data from single stations, inadequate performance was observed for multiple stations and they required 5-10 $\mathrm{min}$ to update the VSL. To provide a quick reaction to the rapidly changing traffic conditions, the VSL system should monitor variations in real time. Hence, a VSL update time of more than $5 \mathrm{~min}$ is expected to cause other traffic problems. Hence, the RVSL system was proposed $[7,8]$.

The JVSL is one of real-time VSL algorithms based on the interworking model between stations on urban freeways. Stations are installed on the freeway with an interval of 1-2 km and collect the traffic information. The JVSL algorithm determines the VSL value based on the information such as speed, density, and traffic volumes. In addition, to quickly react to variations in traffic conditions, the system collects traffic information at $30 \mathrm{~s}$ intervals and calculates the VSL value. The JVSL is composed of four stages. As shown in Figure 1, first, the bottleneck station (BS) where the traffic congestion occurred and its location are identified. Second, based on this information of BS, the VSS location is examined, which represents the tail station of the traffic congestion area, as shown in (Figure 1 (2). Third, based on the VSS, this system determines the number of stations controlled by the VSL (Figure 1 (3)). Lastly, this displays the speed value calculated at each station in the DMS. For several years, the JVSL algorithm has been studied on urban freeways based on the real-time traffic data from multiple stations. However, it showed several problems, which caused malfunctions in the algorithm at various stages of the calculations. In this study, to examine the limitations of the JVSL method, we analyze each stage of the process.

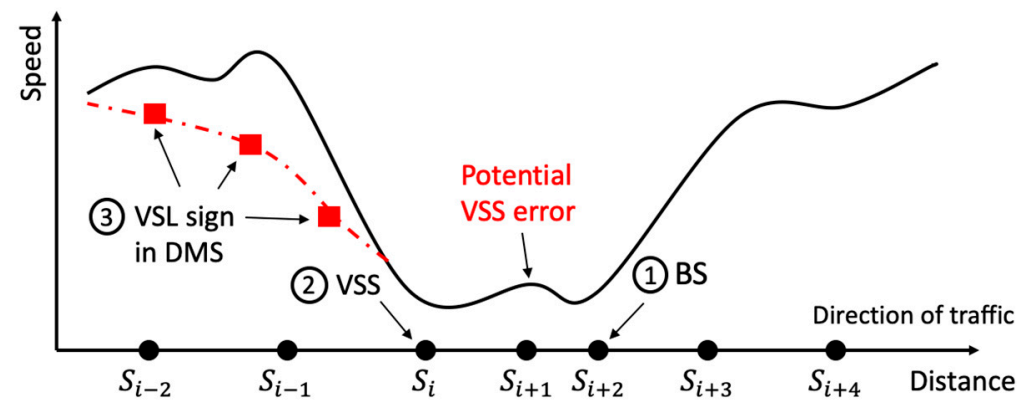

Figure 1. Schematic diagram showing the variable speed limit (VSL) algorithm of the real-time variable speed limit (RVSL) system.

\subsection{Bottleneck Station (BS) Detection}

As shown in Figure 1, to detect the VSS, the JVSL searches the whole area suffering traffic congestion. First, this detects and identifies the start point (start location) of traffic congestion as the BS (Figure 1). This calculates the speed difference between two stations for a period of $1.5 \mathrm{~min}$ in order to identify the BS. However, since the JVSL algorithm selects the BS using only one simple parameter (speed), it cannot quickly react to ever-changing traffic conditions. These critical points cause many traffic control problems for the ITS.

Figure 2 shows three types of speed graphs being operated by the JVSL system in a particular zone, where the traffic speed (red line) are shown at locations of stations. Calculated VSL speeds are shown in blue circles, and displayed VSL speed is adjusted to be displayed in units of $5 \mathrm{~km} / \mathrm{h}$, which are shown in black circles. In addition, the VSS location and traffic congested section are identified. The horizontal axis denotes the stations ( $2.5 \mathrm{~km}$ spacing), while the vertical line shows the speed of each station. The VSS is the selected station connected to the red solid line. For example, in Figure 2a, S7 is the VSS. These figures highlight three problem areas from the JVSL. 
(a)

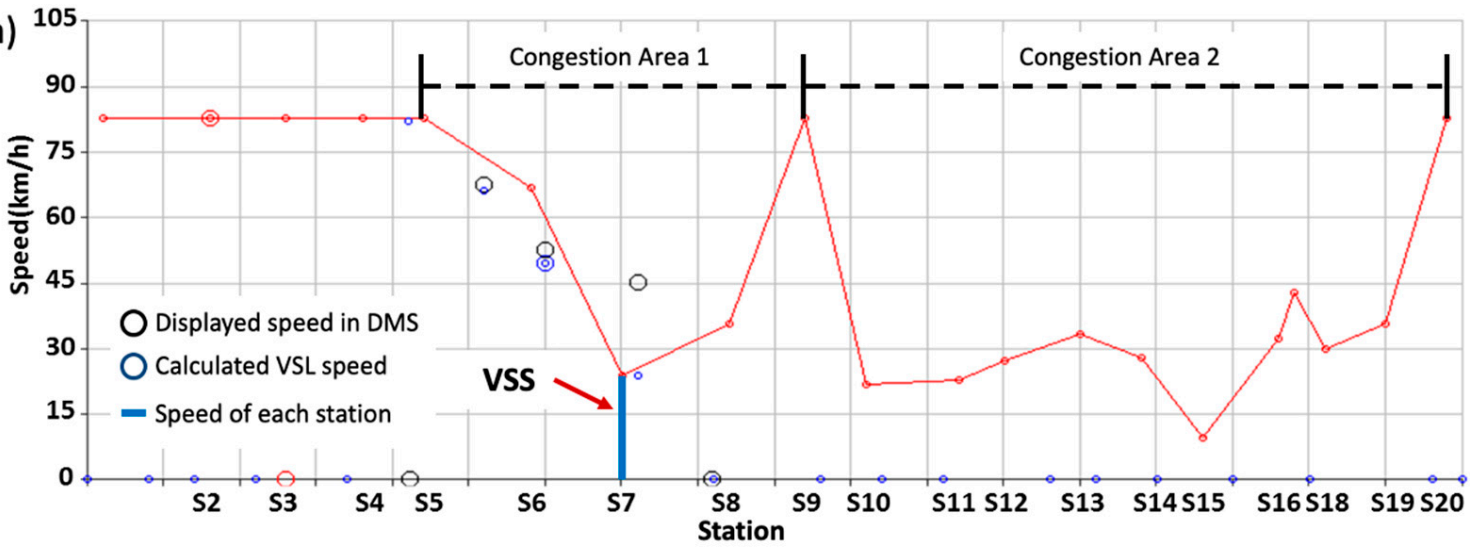

(b)

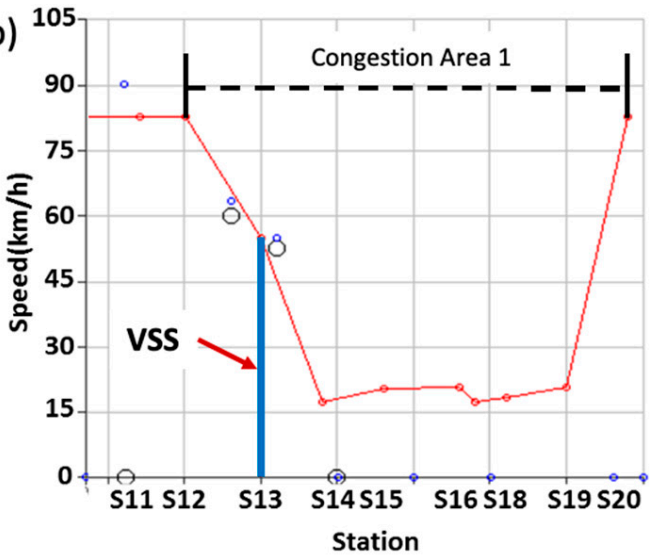

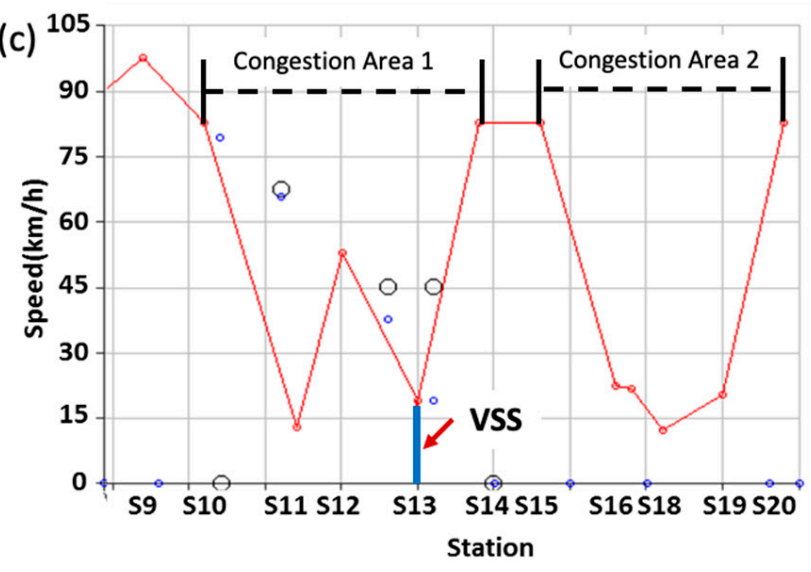

Figure 2. Example graphs of incorrect Variable Speed Limit Start Station (VSS) decision in Jo et al. (JVSL) algorithm [7]. (a) and (c) are scenarios where only 1 VSS is selected for 2 congestion zones. (b) is a scenario where the wrong VSS location is determined. Each graph includes speed (red line) of each station in the congestion section of the freeway.

The example cases in Figure 2a,c include two types of congestion areas, which has low speed flow under $30 \mathrm{~km} / \mathrm{h}$. In fact, when the congestion began, these contained only one congestion area, but, over time, it was changed to two congestion areas. Since the JVSL cannot anticipate this variation in traffic flow in real time, only one VSS (congestion area 1) was selected in each case. However, after $1.5 \mathrm{~min}$, analysis of the speed difference is performed again, and the JVSL can select a different VSS in the congestion area 2 . However, since urban freeways have rapidly varying traffic conditions, this slow reaction capacity of the current JVSL causes safety problems on the road.

In this study, we propose a new method for rapidly detecting the VSS position. The proposed method searches for locations that we expect to be the end of congestion to detect the correct VSS location quickly instead of identifying the full location suffering traffic congestion (Section 3.2.2). In addition, the proposed method can quickly detect the VSS from the predicted locations and can rapidly react to real-time variations in the traffic conditions (Section 3.2.3).

\subsection{VSL Start Station (VSS) Decision}

To detect the VSS, as shown in Figure 1, the JVSL searches the speed (U) of congestion sections from the BS. While searching the process, if the selected station satisfies Equation (1), that station is designated as VSS. $U$ denotes the speed. $S$ denotes the station. $i$ denotes the station number. $t$ denotes the time. Minimum VSL is the lowest speed value to control the vehicle speed in the VSL system. Most of the algorithms varying from studies are set to $30 \mathrm{~km} / \mathrm{h} \mathrm{[4]}$.

$$
\text { If } U_{i-1}-U_{i}>\text { threshold then } V S S=S_{i}
$$


where

$$
\begin{gathered}
\text { If } U_{i-1}>\text { Minimum } V S L_{t} \text { then threshold }=\frac{16 \mathrm{~km}}{h} \\
\text { If } U_{i-1} \leq \text { Minimum } V S L_{t} \text { then threshold = Minimum } V S L_{t-1}
\end{gathered}
$$

JVSL decides the VSS using the speed difference between the current station $\left(U_{i}\right)$ and the next station $\left(U_{i-1}\right)$, where the threshold value is $16 \mathrm{~km} / \mathrm{h}$, as shown in Equation (1). However, since the traffic flow conditions on the road are unpredictable, if an unproven fixed value is used as the threshold value, inaccurate VSS values could be chosen.

First, even in the congested section, the speed difference could be over the threshold value. For example, the speed of S12 (Station 12) is in the congestion area 1 of Figure 2. The VSS in which the VSL should operate is S11 in the congestion area, but S13 is selected as the VSS. Figure 2b also selects the wrong S13 as the VSS rather than S14. Therefore, this system displays an inappropriately high VSL value in the wrong place. Inaccurate VSS decisions lead to problems in the VSL calculation process.

Second, as shown in Figure 2a,c, the VSS cannot be decided from congestion area 2 because the VSL system is suspended for unknown issues (Figure 2a) or spends plenty of time determining and creating the new VSL. The VSL should be constant over the congested section and vehicles entering this region should be continuously controlled. If the VSL system is suspended in the middle of congestion control or is re-started, it can have a severe negative impact on traffic flow.

Our proposed method (Section 3.2.2) addresses the inaccurate VSS decisions made by the JVSL by using both the speed and acceleration from real traffic data to predict possible VSS values. Lastly, an accurate VSS is determined and used to control the congestion areas.

\subsection{Number of Control Stations and VSL Calculation}

The JVSL calculates the number of stations controlled by the VSL. First, by comparing the fixed speed limit with the real traffic speed at the VSS, the scope of controlled stations is designated. If the speed of the VSS is higher than the speed limit, as the difference between the speed decreases, the scope of VSS control also decreases. Second, when a shockwave is calculated, the total congestion situation on the road should be examined. Lastly, the number of control stations is decided using the VSL system.

The JVSL establishes the number of control stations with a minimum of two and a maximum of three. If many stations are controlled by the VSL, the travel time will increase. Hence, an upper limit is set. Since the distance between stations is generally $0.3-2 \mathrm{~km}$, it is acceptable to determine the scope of VSL control, according to the number of stations. However, in this case, the maximum distance over which a reduced speed is applied can reach up to $6 \mathrm{~km}$ and greatly affect the total travel time. Therefore, it has been proposed that the maximum distance over which the VSL is applied is $3.2 \mathrm{~km} \mathrm{[7].}$

As mentioned above, the VSL calculation in the JVSL depends on the number of control stations, where the location and speed of the VSL are calculated using strategy 1,2, and 3. In order to provide accurate VSL control, the DMS location should be included in the process of calculating the VSL. However, JVSL calculates VSL speed based on location of station and display it in the DMS, which is in a completely different location from the station. These are caused to display an incorrect speed limit to the vehicles entering to the congestion area and reach more traffic accidents than when the VSL is not in operation.

In Section 3.3, based on the VSS, the moving time, speed, and acceleration to downward DMS within $3.2 \mathrm{~km}$ are analyzed. The optimal VSL distance and value are selected, along with a VSL value for each DMS. Using this approach, the new VSL system operates based on the DMS instead of the stations, while the vehicle speed can be controlled within the optimal VSL distance.

\section{Enhanced Variable Speed Limits (EVSL)}

VSL systems can decrease the risk of accidents by detecting areas suffering traffic congestion on urban freeways and applying speed limits on those sections. When high speed vehicles approach those with lower speed, the accident rate increases significantly. Hence, the VSL system detects the sections 
with the greatest speed differences between vehicles on the freeway and initiates speed control. It is a goal of VSL systems that total travel times are not significantly decreased when the speed reduction is applied on a freeway. To accomplish this, it is necessary to apply an appropriate speed over an accurate VSL operation area.

In Section 2, the existing JVSL method was introduced and its limitations were discussed.

- The method for selecting the congestion area is too simple. It does not exploit enough traffic data to allow it to rapidly react to the real traffic conditions, which results in inaccurate congested sections and VSS detection.

- Method for determining the VSS using only speed data is too inaccurate and simple. Inaccurate VSS decisions lead to problems in the VSL calculation process that generates the wrong speed limit or system suspend.

- Method for adjusting the maximum VSL distance risks delaying the total travel time. The maximum distance over which a reduced speed is applied can reach up to $6 \mathrm{~km}$ and greatly affect the total travel time.

- Method for calculating the VSL value of each station is based on the location of the VSS instead of DMS. Since the stations and DMS are in different areas, an accurate VSL value cannot be displayed on each DMS device.

Since this method has some critical problems mentioned above, it is impossible to operate the JVSL algorithm to the real urban road for vehicle safety. Therefore, in this section, we aim to apply a new algorithm to the freeway after solving them.

The enhanced VSL algorithm proposed in this case is based on selection of both the DMS and station and is composed of three steps, as shown in Figure 3. First, it calculates the moving average speed to calculate the speed for each station. Second, the VSS is decided based on a new method. Third, the VSL is calculated based on both the location of the VSS and DMS. Lastly, the calculated VSL values are applied to the relevant DMSs. These steps are discussed in more detail in the following sections.

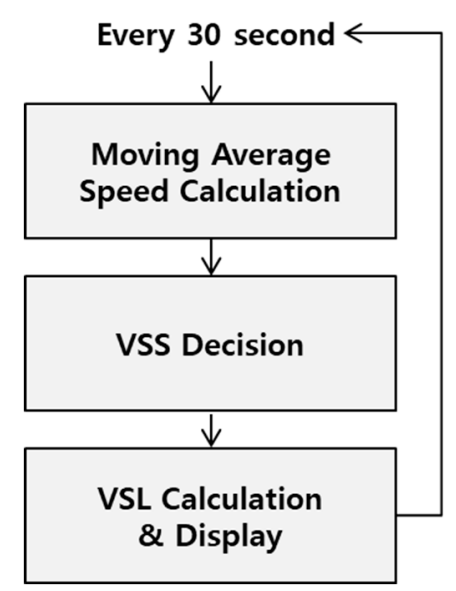

Figure 3. Flow chart of the enhanced variable speed limit (EVSL) algorithm.

\subsection{Moving Average Speed Calculation}

The proposed algorithm quickly reacts to the actual traffic conditions on the freeway. To achieve this, the algorithm takes traffic data every $30 \mathrm{~s}$ from each station and updates the VSL based on recent traffic information. The process of collecting traffic information by the traffic control system to use data in VSL is as follows.

- The traffic detection management devices installed at each station on the freeway collects vehicle data from sensors. 
- The collected data is transmitted to the central server every $30 \mathrm{~s}$ including traffic volume, percent of Occupancy, average speed, and density calculated from each management device.

- The proposed algorithm is performed every $30 \mathrm{~s}$ using vehicle information recorded in a central database.

We obtain the average speed $(u)$, density $(k)$, and volume $(q)$ for $30 \mathrm{~s}$ from the central database. Figure 4 shows the parabolic relationship between the traffic flow (volume) and density [12]. This figure shows that, as the traffic flow increases, the values of flow and density also increase. In addition, the accuracy of speed increases, since the number of vehicles that can be referred to rises as the flow grows.

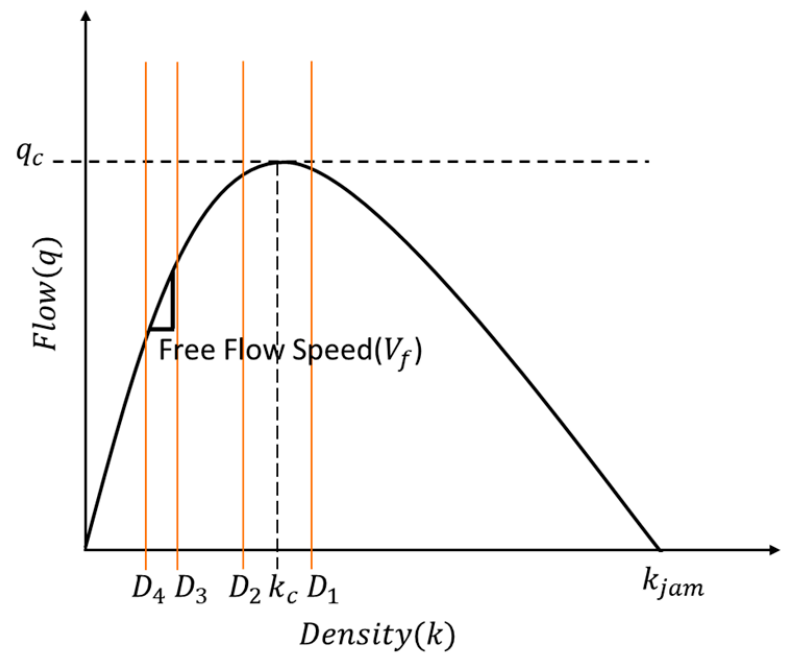

Figure 4. Relation between density and flow.

However, after the maximum flow value is reached, the density value decreases. We realize that traffic congestion begins at this position. We denote this position as the critical density $\left(k_{c}\right)$ at which the road is occupied by the maximum density of vehicles possible. After $k_{c}$, although the density value increases, the traffic flow begins to decrease because traffic congestion results in low vehicle speed.

If the density exceeds $k_{c}$ and reaches jam density $\left(k_{j a m}\right)$, the flow value is 0 . This means that traffic has stopped. From this observation, we define three traffic states [12]: very low flow, free flow $\left(k<k_{c}\right)$, capacity flow $\left(k=k_{c}\right)$, and congestion flow $\left(k>k_{c}\right)$. Therefore, as the density approaches zero, the number of vehicles passing through the sensor decrease, and the accuracy of the speed also decreases. In this case, we can assume the cases where the speed data could be zero when collecting traffic data from sensors every $30 \mathrm{~s}$. There are two cases where the speed is zero. First, there is no vehicle passing the station in the period of $30 \mathrm{~s}$, and the speed and density value are zero. In this case, the proposed algorithm determines the station as an area where the vehicle does not pass, sets the speed of the station to the speed limit $(100 \mathrm{~km} / \mathrm{h})$, and does not select the VSL operating zone. Second, the density reaches jam density $\left(k_{j a m}\right)$ as mentioned in the above paragraph. In this case, we set the speed value considering Equations (2) and (8).

VSL aim to ensure traffic safety and reduce traffic congestion in high traffic environments. Therefore, VSL will tend to be operated at free flow $\left(k<k_{c}\right)$ or capacity flow $\left(k=k_{c}\right)$, and, in this area, the probability of an error calculating the wrong speed is less than low flow and traffic jam, since the speed is calculated using sufficient vehicle data. Basically, the proposed algorithm basically uses a moving average speed. If the road state has a constant speed (uniformly accelerated motion) for $1 \mathrm{~min} 30 \mathrm{~s}$, the sensors constantly measure a constant speed value and we can get relatively high reliability speed data from them. Therefore, the average data for $1 \mathrm{~min}$ (two periods: $30 \mathrm{~s} \times 2$ ) is used as speed data in the general case, as shown in Equation (2). 
However, it may be difficult to obtain accurate speed data if the speed trend of the station has not been constant such as the lowest volume capacity (lowest vehicle density and highest speed) and the congestion area (highest vehicle density and lowest speed: $k_{\text {jam }}$ ) due to low reliability of the sensors. Especially, the sensor cannot measure the accurate data in the congestion area because performance of the vision detector is poor at low speeds and the loop detector also has low speed measurement accuracy at high density traffic flow due to sensor characteristics $[13,14]$. Therefore, the algorithm finds a stable moving average interval (MAI) based on road conditions after deciding whether the measurement area is congested or not based on the density of vehicles, as shown in window size 2 of Equation (2).

$$
M A I= \begin{cases}2, & u_{t-2, i}<u_{t-1, i}<u_{t, i} \text { or } u_{t-2, i}>u_{t-1, i}>u_{t, i} \text { or } u_{t-2, i}=u_{t-1, i}=u_{t, i} \\ 6, & 35 \leq k \\ 4, & 35>k \geq 25 \\ 3, & 25>k \geq 15 \\ 4, & 15>k \geq 10 \\ 6, & 10>k\end{cases}
$$

To classify the window size of the average speed based on the density at the congested area, we classify each density area based on average $k_{c}$ of the freeway where the VSL is operated in. First, $k_{c}$ of each station is obtained from Equation (3). It is calculated by the Green Shield Model using the maximum traffic flow $\left(Q_{c}\right)$ and free flow speed $\left(V_{f}\right)$ [15].

$$
k_{c}=\frac{Q_{c}}{v_{f}}
$$

$Q_{c}$ is obtained from Equation (4). $k_{j a m}$ refers to the density of traffic congestion. To calculate Equations (3) and (4), $V_{f}$ and $k_{j a m}$ should be calculated first. They can be obtained from real road data. However, it is difficult to calculate these two values accurately. Therefore, we calculate their approximate values from Equations (5) and (6) based on the speed $(v)$ and density $(k)$ values obtained for the corresponding road. However, the $v$ and $k$ values vary depending on whether the traffic state is smooth or congested. In this study, traffic data are collected for a real road, and the $k$ and $k_{j a m}$ values are obtained from Equations (5)-(7).

$$
\begin{gathered}
Q_{c}=\frac{v_{f} \times k_{j a m}}{4} \\
k_{j a m}=\frac{v_{f}}{|b|} \\
v_{f}=\bar{v}-b \bar{k} \\
b=\frac{\sum\left(k_{i}-\bar{k}\right)\left(v_{i}-\bar{v}\right)}{\sum\left(k_{i}-k\right)^{2}}
\end{gathered}
$$

We set the window size for calculating the moving average of the speed according to the reliability of the sensor if the trend of the speed for $1 \mathrm{~min}$ and $30 \mathrm{~s}$ is not constant. The window size is set based on the density since the reliability of the sensor is determined by the number of vehicles passing through the sensor. First, the density section, $k_{c}$, where congestion begins is determined. Each station on the road has a different $k_{c}$ value. Figure 5 shows the average speed and q-k graph for the congestion area of the Seoul Outer Ring freeway from January 2016 to January 2017. Referring to q-k scatter graph, $k_{c}$ distribution of each station can be seen to have a value of about \pm 5 , based on the average density value 30. Therefore, we determined the interval of $k_{c}$ in Figure 4 as $25\left(D_{1}\right)-35\left(D_{2}\right)$ based on empirical data in order to generalize the $k_{c}$ values of all stations. Second, in the case of a highly reliable free flow speed area that includes a large number of vehicles as in Figure 5, the density value for selecting the window 
size is determined according to the flow $(q)$ value. In this paper, we set the free flow speed area from $q_{c}$ to a minimum value of flow by referring to the q-k scatter values in Figure 5 . Therefore, $D_{4}$ in Figure 4 is determined as the density value of 10 at the minimum value position of the free flow area. Third, $D_{3}$ for setting the confidence area in the free flow uses the value of $75 \%$ from the reference value of $D_{4}$ [15]. In this paper, we use the value of $D_{3}$ as 15. Lastly, the window size is determined as in Equation (2). The lowest density and highest density areas with very low vehicle information are 6 ( $3 \mathrm{~min}$ ), the low density and high-density areas are 4 ( $2 \mathrm{~min})$, and the free flow speed area is $3(1 \mathrm{~min} 30 \mathrm{~s})$.

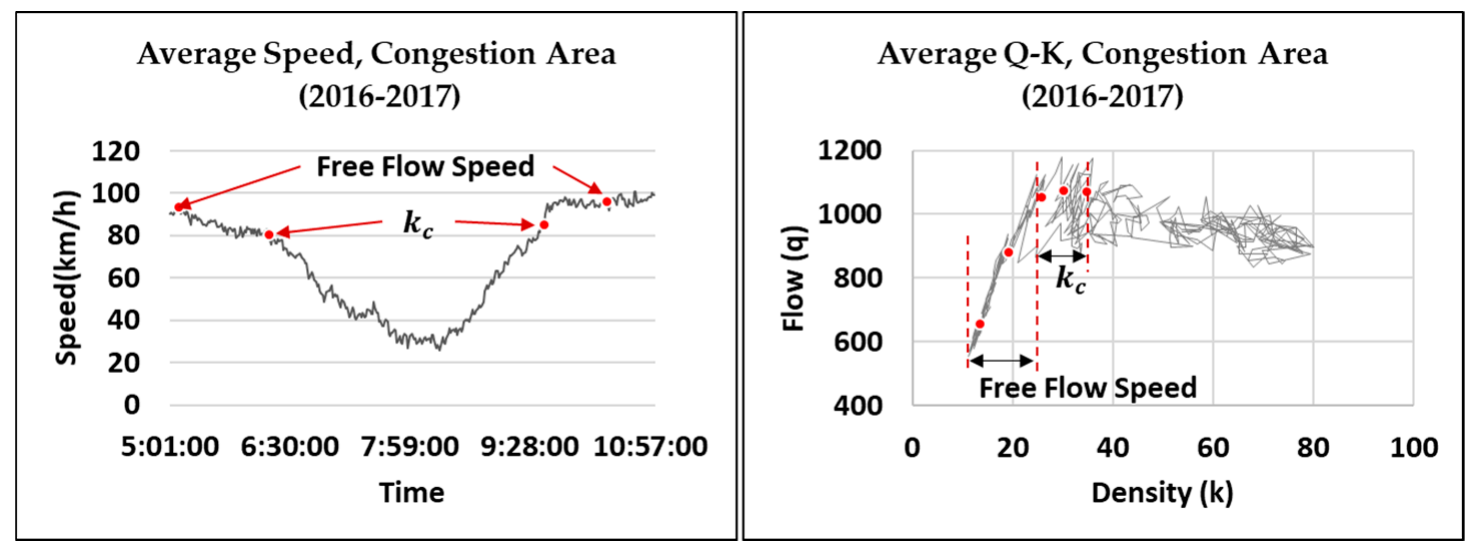

Figure 5. The average speed and $\mathrm{q}-\mathrm{k}$ graph for the congestion area of the Seoul Outer Ring freeway from January 2016 to January 2017.

The moving average speed is calculated using Equation (8). $u$ denotes the speed, $t$ denotes the time, $i$ denotes the station number, and $k$ denotes the density.

$$
u_{\text {avg }}(k m / h)=\frac{\sum_{n=0}^{M A I-1} u_{t-n, i}}{M A I}
$$

\subsection{VSL Start Station (VSS) Decision}

The station at the start of the congestion area is designated as the VSS, and the VSL system controls the speed of vehicles from this point (where the high-speed traffic meets the slower vehicles), as shown in Figure 1. Based on the selected VSS, the VSL value is established for each station, as shown in Figure 1. Therefore, an accurate decision of the VSS is necessary to ensure stable performance.

Our EVSL algorithm provides a more accurate and efficient manner of determining the VSS location compared to the standard RVSL system. This is achieved by using the vehicle acceleration between stations to assist in the detection of pVSS locations and selection of a final VSS. High acceleration values are usually observed at pVSS locations.

The algorithm analyzes past traffic data for the pVSS locations and compares them to the real acceleration values in order to identify locations with significant variations in traffic flow. Generally, at the start of the congested region, the traffic flow changes abruptly and large differences in the speed between stations are observed. Therefore, the predicted VSS locations can be detected accurately. Lastly, this system selects the final VSS after a merging operation is performed on overlapping sections, which are assumed to be a single congested section among the pVSS locations.

\subsubsection{Acceleration Calculation}

The pVSS locations are selected as described in the previous section. When acceleration values between stations are calculated, accurate speed differences can be predicted for each station. Figure 6 shows the method for calculating the acceleration at each station. Basically, EVSL calculates an acceleration using the distance and speed between station $S_{i}$ and the preceding station $S_{i-1}$, as shown 
in Figure 6, where Equation (9) shows the corresponding acceleration equation. $u$ denotes the speed, $i$ denotes the station number, and $d$ denotes the distance between stations.

$$
\text { Acceleration of } \operatorname{Station}_{i}\left(\mathrm{~km} / \mathrm{h}^{2}\right)=\frac{u_{i}{ }^{2}-u_{i-1}{ }^{2}}{2 \times d}
$$

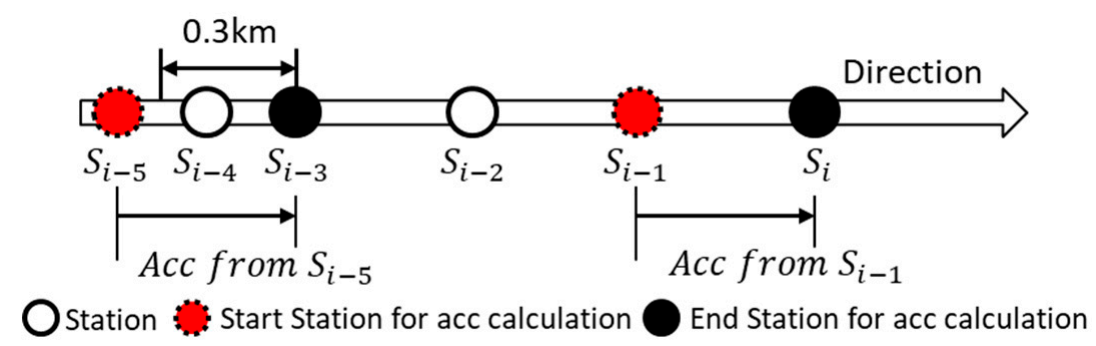

Figure 6. Schematic diagram showing the method for calculating acceleration at each station.

If the distance between two stations is less than $300 \mathrm{~m}$, when vehicles are travelling over $80 \mathrm{~km} / \mathrm{h}$, the speed difference between two stations is too small to detect. Hence, we calculate the acceleration over a minimum distance of $300 \mathrm{~m}$. As shown in Figure 6, the downstream station $\left(S_{i-4}\right)$ installed within $300 \mathrm{~m}$ from the upstream $\left(S_{i-3}\right)$ station is merged with the upstream station $\left(S_{i-3}\right)$ and excluded from the acceleration calculation and pVSS determination. Therefore, the acceleration for $S_{i-3}$ is calculated with respect to $S_{i-5}$ instead of $S_{i-4}$.

\subsubsection{Preliminary VSS (pVSS) Decision}

First, our EVSL algorithm selects candidate VSSs that are considered to be tail portions of congestion areas to determine an accurate VSS location. In generally, VSS is the tail portion of the congestion area. If a vehicle approaches the VSS, its speed should rapidly decrease. Therefore, as shown in Figure 7, we can estimate that two areas (between $S_{i-2}$ and $S_{i-3}$ and between $S_{i-4}$ and $S_{i-5}$ ) are the pVSS, which is a high deceleration congestion estimated area. Hence, $S_{i-2}$ and $S_{i-4}$ are selected as pVSS locations following the criteria given by Equations (10) and (11), which use the acceleration and speed values between the current and previous stations.

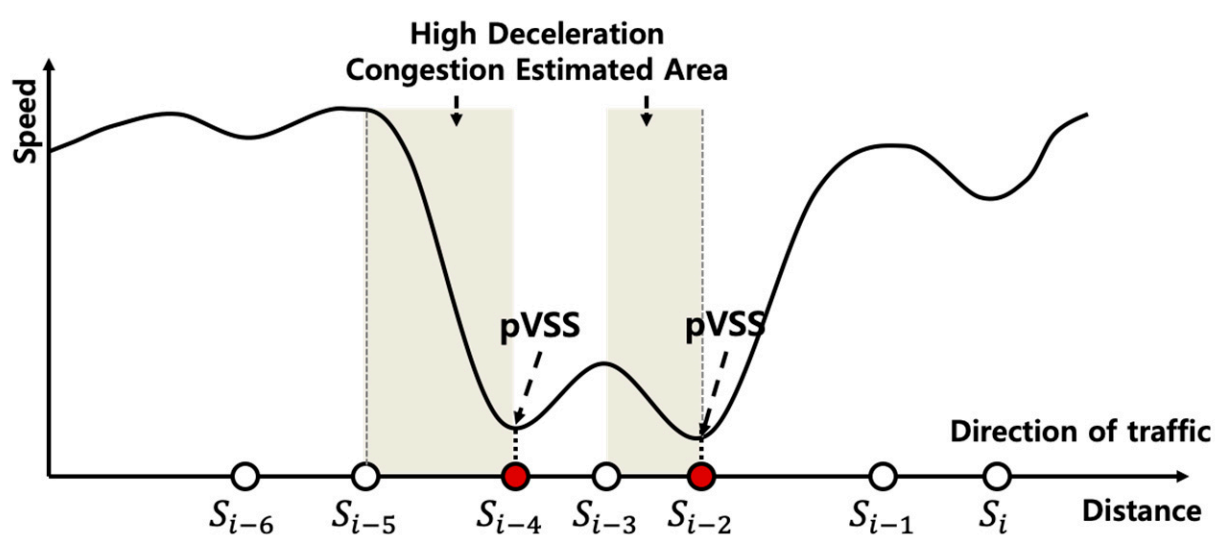

Figure 7. Schematic diagram showing the method for determining the preliminary VSL start station (pVSS) locations from the station location and speed.

To select the pVSS, the threshold value of acceleration and speed was investigated [16]. This approach is based on reports of accidents occurring on the I-35W freeway in Minnesota, USA. We judged that there would be no problem even if the data investigated in Reference [16] was used because the road environments of the Seoul outer ring freeway composed of the experimental environment and the I-35W were similar. This includes the number of lanes (I-35W: 3-5, Seoul outer 
ring: 3-5), speed limit (I-35W: $104 \mathrm{~km} / \mathrm{h}$, Seoul outer ring: $100 \mathrm{~km} / \mathrm{h}$ ), and more. Figure 8 shows the distribution of acceleration and speed for the accidents occurring for 2009.9-2009.12 on the I-35W freeway, which has a normal speed limit of $104 \mathrm{~km} / \mathrm{h}(65 \mathrm{mile} / \mathrm{h})$. It can be seen that $60 \%$ of the accidents occurred below $90 \mathrm{~km} / \mathrm{h}(55 \mathrm{mile} / \mathrm{h})$ and below acceleration values of $-2400 \mathrm{~km} / \mathrm{h}^{2}\left(-1500 \mathrm{mile} / \mathrm{h}^{2 \wedge} 2\right)$. In this case, when the current station $\left(S_{t, i}\right)$ is not the VSS at $\mathrm{t}-1$, if the speed of $S_{t, i}$ is $<90 \mathrm{~km} / \mathrm{h}$ and the acceleration is below $-2400 \mathrm{~km} / \mathrm{h}^{2}, S_{t, i}$ could be selected as a pVSS in Equation (10). $S$ denotes the Station, $t$ denotes the time, $i$ denotes the station number, $u$ denotes the speed, and $a$ denotes the acceleration.

$$
S_{t, i}=p V S S, \text { if } u_{(t-2, t-1, t), i} \leq 90 \mathrm{~km} / \mathrm{h} \text { and } a_{(t-2, t-1, t), i-1} \leq-2400 \mathrm{~km} / \mathrm{h}^{2}
$$

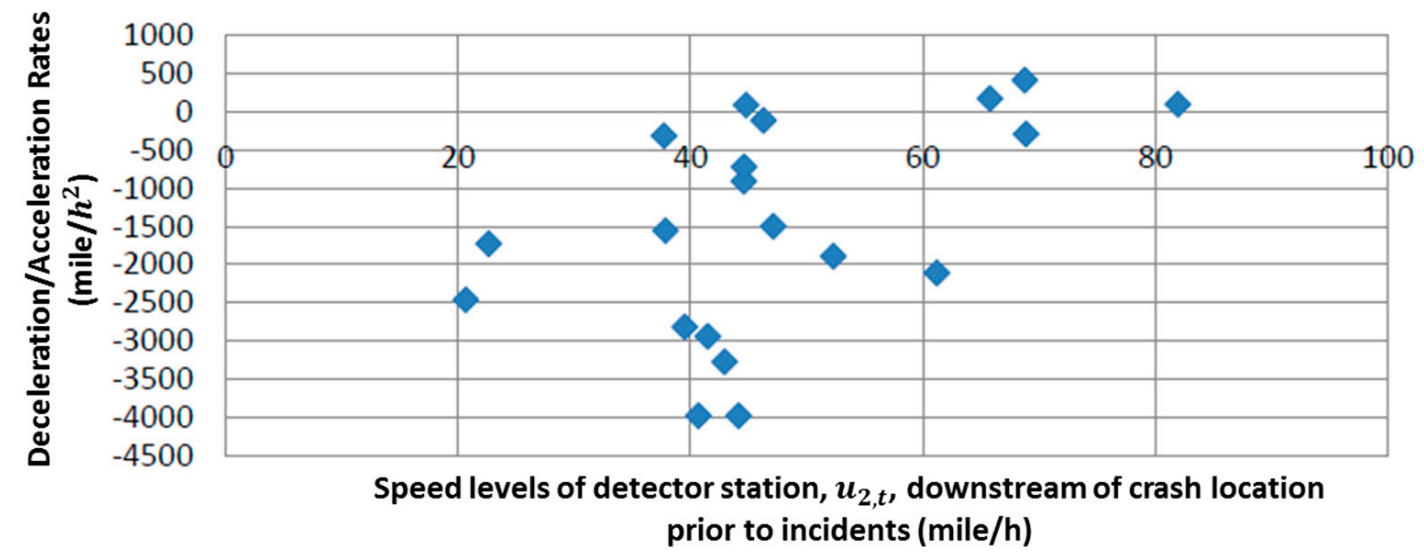

Figure 8. Measured accelerations of traffic flow prior to accidents on the I-35W, Minnesota, USA (2009).

In addition, when $S_{t, i}$ was assigned as the VSS at $\mathrm{t}-1$, if it satisfies Equation (11), it could continuously be assigned as a pVSS.

$$
S_{t, i}=p V S S, \text { if } a_{(t-2, t-1, t), i-1} \leq-1200 \mathrm{~km} / \mathrm{h}^{2}
$$

In our EVSL algorithm, to reduce the error of VSS calculation arising from the short update period and allow stable selection of the pVSS, if a station satisfies the conditions of Equation (10) or (11) for $1.5 \mathrm{~min}$, it qualifies as a pVSS.

\subsubsection{VSS Decision}

The VSS is finally selected from the group of pVSS. Figure 9 shows the congestion area, locations of pVSS, and the method for determining the VSS. In Figure 9a, there are two pVSSs that can be final VSS in the congestion area. However, if the VSS is chosen in the congestion area as shown by the pVSS, $S_{i-2}$ in Figure 9a, this may worsen the congestion than before. In addition, since the speed of the congestion area is already low, application of a VSL in this area is not necessary. Therefore, such unnecessary pVSS locations should be eliminated and an accurate VSS is required to control the speed of the vehicles approaching the congested area. 

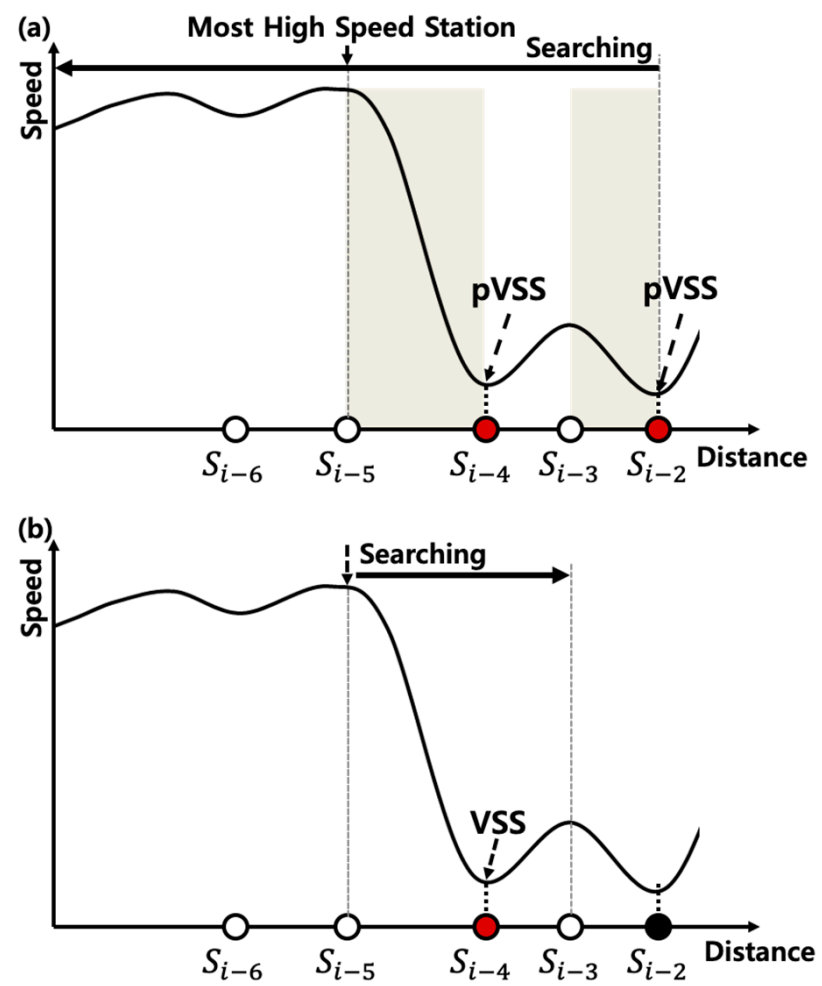

Figure 9. Schematic diagram showing the method for determining the variable speed limit start station (VSS). (a) The location of pVSS and upward search in the opposite direction of traffic flow to find the scope of VSL. (b) downward searching to select VSS.

First, to achieve this, upward searching opposite of the direction of traffic flow is performed from the first downstream pVSS location. Searching proceeds until the condition of Equation (12) is satisfied and part of the congestion area is defined by locating the first station that is not affected by traffic congestion. We used a threshold value of congestion of $40 \mathrm{~km} / \mathrm{h}$ following the definition of the Korea Expressway Corporation [17]. $S$ denotes the Station, $t$ denotes the time, $i$ denotes the station number, $u$ denotes the speed, and $a$ denotes the acceleration.

$$
S_{t, i}=\text { End of Congestion, if } a_{t, i}>0 \mathrm{~km} / \mathrm{h}^{2} \text { and } u_{\mathrm{t}, \mathrm{i}} \geq 40 \mathrm{~km} / \mathrm{h}
$$

Next, as shown in Figure 9b, downward searching is performed from the station selected by Equation (12) until the condition of Equation (13) is satisfied. As shown in the figure, the search operation is performed up to the station near the congestion area.

$$
\text { Downstream Searching Stop Condition, if } a_{t, i}>0 \mathrm{~km} / \mathrm{h}^{2}
$$

Lastly, while the search process of Equation (13) is performed, the station satisfying the condition shown in Equation (14) is updated as the VSS. The VSS is updated until the search process is finished, which results in the pVSS near the end of the congestion area being designated as the VSS.

$$
S_{t, i}=V S S, \text { if } a_{t, i} \leq-1200 \mathrm{~km} / \mathrm{h}^{2}
$$

\subsection{VSL Calculation and Display}

The VSL system displays the corrected speed for each section of road via DMS installed on the freeways. To improve on the existing JVSL algorithm that assumed that both the DMS and station were at the same location, our new algorithm first defines the location of the DMS and VSL values corresponding to specific DMS calculated based on the VSS. This results in more suitable speed being 
displayed on each DMS, where the vehicles entering the congestion area show a reduced speed on the DMS located upstream from the VSS. Figure 10 shows the relationship between the VSS and DMS, where the empty circles denote the station and filled circle denote the VSS. As shown in Figure 10a, the VSS is connected to all DMS systems located upstream, which defines the VSL distance. As shown in Figure 10b, if the current VSS and the upstream VSS connect with the same DMS. The lower VSS has priority.

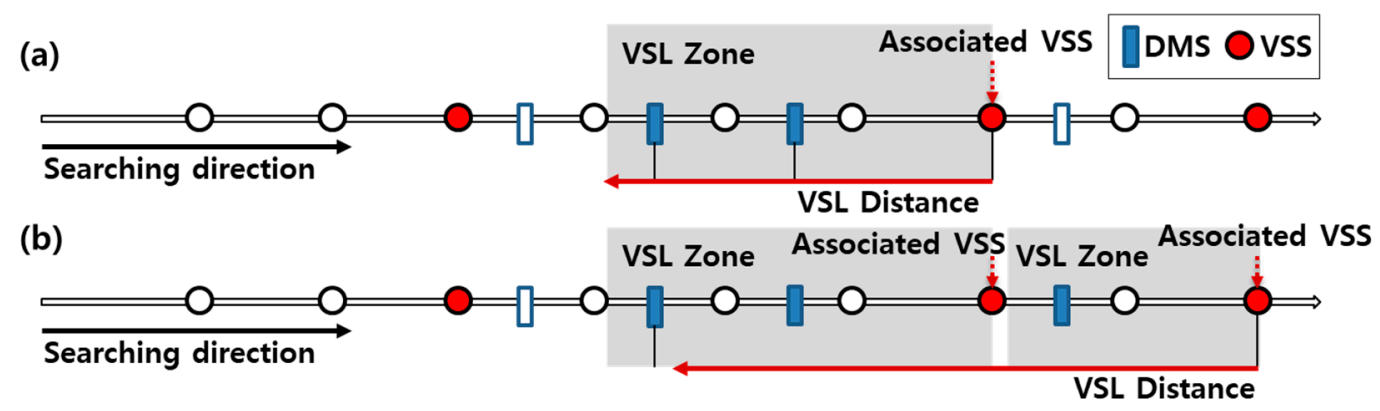

Figure 10. Schematic diagram showing the relationship between the dynamic message sign (DMS) and VSL start station (VSS). (a) A single VSS scenario for associating with DMS, (b) Multiple VSS scenario for associating with DMS.

As shown in Figure 11, the VSL distance is the total distance from the VSS over which the VSL is applied, which was limited to $3.2 \mathrm{~km}$ in our algorithm in an attempt to not affect the travel time of vehicles within a travel distance [4].

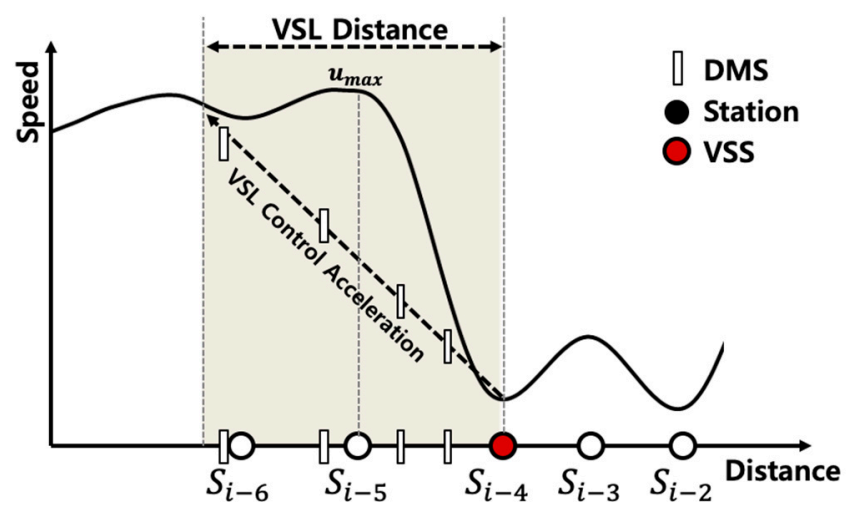

Figure 11. Schematic diagram showing the method for controlling the variable speed limit (VSL).

A new equation for the VSL distance is proposed, which is based on the speed, moving time, and acceleration, as shown in Equation (15). Based on the current speed at the VSS, if VSL control acceleration $\left(a_{t, i}: \mathrm{VCA}\right)$ is known, the total distance to reach the speed limit $\left(u_{\text {limit }, i}\right)$ can be calculated. $u$ denotes the speed, limit denotes the speed limit, $i$ denotes the station number, $t$ denotes the time, and $a$ denotes the acceleration.

$$
\operatorname{VSL} \operatorname{Distance}(k m)=\frac{u_{\text {limit, },{ }^{2}-u_{t, i}{ }^{2}}}{2 \times \alpha_{t, i}}
$$

As shown in Figure 11, the VCA is used to calculate and decide the VSL distance and speed in each DMS. In previous studies, the acceleration value was fixed as $-2400 \mathrm{~km} / \mathrm{h}^{2}$ [4]. However, the speed and distance vary according to the speed limit and traffic conditions. If the VSL is determined using a fixed acceleration, wrong traffic information could be provided to vehicles on the freeway. Therefore, a new method for calculating the VCA is required, which updates the value in real time. The new equation for the $\operatorname{VCA}\left(a_{t, i}\right)$ is shown by Equation (16), which exploits the speed difference between the 
speed of the current VSS and the station that has the maximum speed within the $3.2 \mathrm{~km}$ VSL distance from current VSS. In addition, this equation uses the travel time $\left(t t_{t, i}\right)$ between $3.2 \mathrm{~km}$ from the VSS to consider the current traffic condition. Lastly, VCA is calculated using travel time from the VSS and the speed difference, which is shown in Equation (16). $u$ denotes the speed, $i$ denotes the station number, $t$ denotes the time, $a$ denotes the acceleration, $d$ denotes the distance, and $\gamma$ denotes the limited station. Equation (17) decides the limit of stations. Since the length of the VSL is limited to $3.2 \mathrm{~km}$, if distance between current VSS and the station is more than $3.2 \mathrm{~km}$, subsequent stations are not applied to the VCA calculation.

$$
\begin{gathered}
\alpha_{t, i}\left(\mathrm{~km} / h^{2}, \mathrm{VCA}\right)=\frac{\operatorname{MAX}\left(u_{t, i}, u_{t, i-1}, \cdots u_{t, i-\gamma}\right)-u_{t, i}}{t t_{t, i} \text { to } i-\gamma} \\
\gamma=i,\left|d_{t, i}-d_{t, v s s}\right|>3.2 \mathrm{~km}
\end{gathered}
$$

Based on VCA, the speed of each DMS connected to the VSS is calculated using Equation (18). To calculate the VSS speed, $\operatorname{VCA}\left(a_{t, i}\right)$ and the distance between VSS and DMS are used, and the final speed of each DMS can be achieved. $u$ denotes the speed, $i$ denotes the station number, $t$ denotes the time, $a_{t, i}$ denotes the VCA, $q$ denotes the DMS, and $L$ denotes the distance between VSS and DMS.

$$
u_{t, q}=\sqrt{u_{t, V S S^{2}+2 \times \alpha_{t, i} \times L}}
$$

The VSL displayed on the DMS has the following restrictions: the minimum speed close to the congested section is $40 \mathrm{~km}$ and the maximum speed is $80 \mathrm{~km}$ (at this value, the vehicle begins to reduce its speed). Since the distance between the DMS locations is included in the calculation, the speed at the DMS location is displayed at $5 \mathrm{~km} / \mathrm{h}$ intervals following the definition of the Korea Expressway Corporation [17].

\section{Experimental Environment for Microscopic Simulation}

The proposed algorithm was tested on sections of an outer ring expressway located in the south of Seoul, Republic of Korea. This is a 25-km section located between Byeollae-IC and West-Hanan-IC (Figure 12) with seven interchanges and two intersections connected to two freeways. This includes a total of 23 stations, which are installed every $400 \mathrm{~m}$ to $2200 \mathrm{~m}$ and have the vision detections to obtain the vehicle information in each lane, as shown in Figure 12. In the experiment, we used the Verkehr In Städten-SIMulationsmodell 6 (VISSIM 6, Karlsruhe, Germany, 2013) simulator supported by the Planung Transport Verkehr (PTV) Corporation [18], which is a micro-simulator that can be implemented and tested for various road environments. To control the traffic flow and VISSIM simulator, we implemented the Korea highway traffic analysis (KHTA) system, which controlled VSSIM and provided similar real road conditions in our experiments [19]. After the simulation was complete, the performance of the VSL algorithm was evaluated using performance metrics. The experiment was executed using the time zone suffering the most severe traffic congestion ( $5 \mathrm{AM}$ to 11 AM). In addition, to simulate the pattern of various vehicles, five types of random seeds (Seed 10, $15,17,20$, and 25) were used to create vehicle patterns. In the simulation, open traffic data provided by the Korea Expressway Corporation were used [20]. In addition, the system was calibrated to tailor it for similar real road environments using the method proposed by Berkeley using the changing vehicle-following behavior value [21].

VISSIM provides the various calibration parameters to control the vehicle behavior and regulation on the road for the simulation. The calibration of parameters is the most important during the simulation process because the vehicles in the simulator behave differently depending on the calibration parameters. Therefore, it is necessary to adjust the vehicle behavior in the simulator similarly to the real traffic flow through the calibration of the simulator parameters. In Figure 12, the bottlenecks of the measurement section are Guri IC, Topyung IC, Gang-il IC, Sang-il IC, and Seo-hanam IC, which are interchanges that many vehicles enter from another urban freeway or highway. 


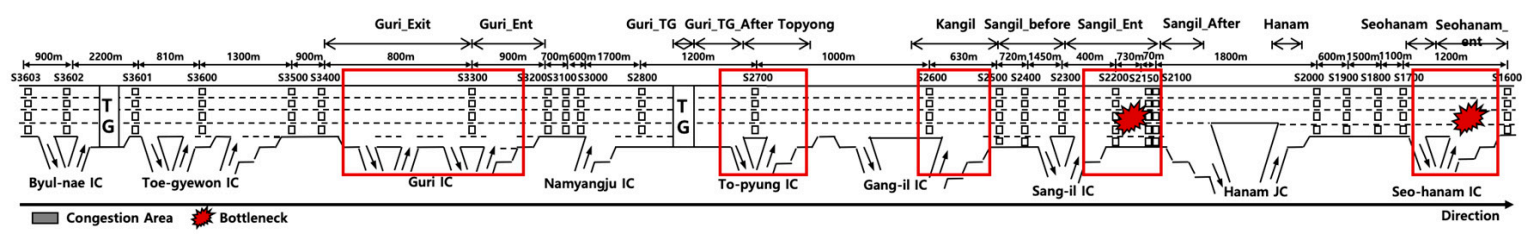

Figure 12. Schematic diagram showing the section of the road (Seoul Outer Ring Road) tested in the simulations.

Tables 1 and 2 are calibration parameters to make the bottlenecks similar to a real traffic road in the simulation environment. Table 1 has calibration parameters for traffic flow control based on the Wiedemann model, which has a model version released in 1974 and 1999 [22,23]. These models have 10 types of calibration parameters, which are named Calibration and Comparison (CC) and classified by distance, speed, acceleration value, and more. We adjusted only three parameters to adjust the traffic flow, which are CC1 (Headway Time), CC8 (Standstill Acceleration), and CC9 (Acceleration at $80 \mathrm{~km} / \mathrm{h}$ ). CC1 is desired headway time between the lead and following vehicles. CC8 is the desired acceleration from standstill. CC9 is the desired acceleration at $80.45 \mathrm{~km} / \mathrm{h}$. Since these are key parameters to affect the traffic flow and make a bottleneck, we use only them to adjust traffic flow of the simulator environment.

Table 1. Calibration parameters (CC1, CC8, CC9) for Traffic Flow Control of Verkehr In Städten SIMulationsmodell (VISSIM).

\begin{tabular}{cccc}
\hline & Parameter for Each Station & \\
\hline Link Type & $\begin{array}{c}\text { CC1 } \\
\text { Headway Time }\end{array}$ & $\begin{array}{c}\text { CC8 } \\
\text { Standstill } \\
\text { Acceleration }\end{array}$ & $\begin{array}{c}\text { CC9 } \\
\text { Acceleration } \\
\text { At } 80 ~ k m / h\end{array}$ \\
\hline Freeway & $1.0 \mathrm{~s}$ & $3.5 \mathrm{~m} / \mathrm{s}^{2}$ & $1.5 \mathrm{~m} / \mathrm{s}^{2}$ \\
Guri Exit & $1.2 \mathrm{~s}$ & $1.28 \mathrm{~m} / \mathrm{s}^{2}$ & $1.5 \mathrm{~m} / \mathrm{s}^{2}$ \\
Guri Entrance & $1.85 \mathrm{~s}$ & $2.28 \mathrm{~m} / \mathrm{s}^{2}$ & $1.5 \mathrm{~m} / \mathrm{s}^{2}$ \\
Guri Toll Gate (TG) & $0.5 \mathrm{~s}$ & $3.28 \mathrm{~m} / \mathrm{s}^{2}$ & $1.8 \mathrm{~m} / \mathrm{s}^{2}$ \\
Guri TG After & $1.0 \mathrm{~s}$ & $4.19 \mathrm{~m} / \mathrm{s}^{2}$ & $1.5 \mathrm{~m} / \mathrm{s}^{2}$ \\
Topyong & $2.3 \mathrm{~s}$ & $1.85 \mathrm{~m} / \mathrm{s}^{2}$ & $1.0 \mathrm{~m} / \mathrm{s}^{2}$ \\
Kangil & $1.75 \mathrm{~s}$ & $2.0 \mathrm{~m} / \mathrm{s}^{2}$ & $1.5 \mathrm{~m} / \mathrm{s}^{2}$ \\
Sangil Before & $1.0 \mathrm{~s}$ & $3.19 \mathrm{~m} / \mathrm{s}^{2}$ & $1.5 \mathrm{~m} / \mathrm{s}^{2}$ \\
Sangil Entrance & $2.4 \mathrm{~s}$ & $2.28 \mathrm{~m} / \mathrm{s}^{2}$ & $1.19 \mathrm{~m} / \mathrm{s}^{2}$ \\
Sangil After & $1.8 \mathrm{~s}$ & $2.58 \mathrm{~m} / \mathrm{s}^{2}$ & $1.19 \mathrm{~m} / \mathrm{s}^{2}$ \\
Hanam & $1.8 \mathrm{~s}$ & $3.19 \mathrm{~m} / \mathrm{s}^{2}$ & $1.5 \mathrm{~m} / \mathrm{s}^{2}$ \\
Seohanam & $2.5 \mathrm{~s}$ & $1.0 \mathrm{~m} / \mathrm{s}^{2}$ & $0.95 \mathrm{~m} / \mathrm{s}^{2}$ \\
Seohanam Entrance & $2.5 \mathrm{~s}$ & $1.5 \mathrm{~m} / \mathrm{s}^{2}$ & $1.0 \mathrm{~m} / \mathrm{s}^{2}$ \\
\hline
\end{tabular}

Table 2. Calibration parameters for lane change control of VISSIM.

\begin{tabular}{|c|c|c|c|}
\hline \multicolumn{4}{|c|}{ Parameters for Lane Change } \\
\hline Necessary Lane Change & $\begin{array}{c}\text { Maximum } \\
\text { Deceleration }\end{array}$ & $-1 \mathrm{~m} / \mathbf{s}^{2}$ per Distance & $\begin{array}{c}\text { Accepted } \\
\text { Deceleration }\end{array}$ \\
\hline Own & $-3.0 \mathrm{~m} / \mathrm{s}^{2}$ & $77.33 \mathrm{~m}$ & $-0.91 \mathrm{~m} / \mathrm{s}^{2}$ \\
\hline Trailing Vehicle & $-3.25 \mathrm{~m} / \mathrm{s}^{2}$ & $70 \mathrm{~m}$ & $-1.19 \mathrm{~m} / \mathrm{s}^{2}$ \\
\hline \multicolumn{2}{|c|}{ Wait Time Before Diffusion } & \multicolumn{2}{|c|}{ Minimum Headway } \\
\hline \multicolumn{2}{|c|}{$23.13 \mathrm{~s}$} & \multicolumn{2}{|c|}{$0.09 \mathrm{~m}$} \\
\hline
\end{tabular}

Table 2 includes calibration parameters to adjust a behavior method for the lane change of the vehicle in a simulator. The ego vehicle and trailing vehicle is affected from the Own vehicle. Maximum deceleration is max deceleration of more than two vehicles and $-1 \mathrm{~m} / \mathrm{s}^{2}$ per distance is preparing 
distance to reduce the speed of each vehicle. An accepted deceleration is the minimum deceleration of each vehicle. Above parameter values are already measured from Shin at al. [24], which simulates the same simulation section. Therefore, we fully follow the measurement values of the calibration parameters obtained above.

\section{Performance Evaluation}

\subsection{VSS Stability Assessment}

A major advantage of VSL is its fast response to sudden events, such as road repair works and vehicle accidents. Since the VSL system enhances road safety, it is an important solution for a traffic environment. In addition, it aims to reduce the total travel time through traffic flow control. Existing RVSL algorithms provide the VSL system, which focuses on traffic control between multiple stations in the freeway. However, they select and use the wrong VSS, which is in the wrong place due to the VSS selection error mentioned in Section 2.2. Hence, this wrong VSS occurs with various errors of the whole VSL system, which cannot control and worsen the traffic flow.

Since the new EVSL was developed to improve accuracy of the VSS selection and reduce propagation of errors in the total VSL system, we compared the VSS detection results using both the JVSL and the EVSL algorithms, as shown in Table 3. Total simulation time is about $6 \mathrm{~h}$ at each random seed, and the error rates in the selection of the VSS location were measured at $30 \mathrm{~s}$ intervals. The error rates were calculated as the VSS locations \pm 2 stations from the position where the correct VSS should be located. The location of correct VSS was set manually by the human before re-simulation.

Table 3. VSL start station (VSS) decision error result.

\begin{tabular}{|c|c|c|c|c|c|c|}
\hline \multirow{3}{*}{$\begin{array}{l}\text { Random } \\
\text { Seed }\end{array}$} & \multicolumn{3}{|c|}{ JVSL } & \multicolumn{3}{|c|}{ EVSL } \\
\hline & Total VSL & Error & Error Rate & Total VSL & Error & Error Rate \\
\hline & \multicolumn{2}{|c|}{ (30 s Interval) } & (Percent) & \multicolumn{2}{|c|}{ (30 s Interval) } & (Percent) \\
\hline SEED 10 & 505 & 112 & $22.2 \%$ & 555 & 1 & $0.2 \%$ \\
\hline SEED 15 & 512 & 121 & $23.6 \%$ & 571 & 0 & $0.0 \%$ \\
\hline SEED 17 & 488 & 97 & $19.9 \%$ & 532 & 0 & $0.4 \%$ \\
\hline SEED 20 & 511 & 109 & $21.3 \%$ & 540 & 1 & $0.2 \%$ \\
\hline SEED 25 & 503 & 85 & $16.9 \%$ & 545 & 0 & $0.0 \%$ \\
\hline Average & 504 & 105 & $21 \%$ & 549 & 1 & $0.1 \%$ \\
\hline
\end{tabular}

In Table 3, the numeric unit of the Total VSL and Error is 'tick,' which has an interval of $30 \mathrm{~s}$. Total VSL refers to the VSL working time among total time (720 ticks) and the error is the error issued time. The experimental results for the five different random seeds used are shown. It can be seen that the JVSL algorithm showed a VSS decision error around every $1 \mathrm{~h}$ (105 ticks/30 s), corresponding to a high error rate of $21 \%$, which has a negative effect on the calculation of the final VSL (in addition to the traffic volume and accident risk). In contrast to the JVSL algorithm, the EVSL algorithm had a very low error rate below $0.5 \%$ due to the selection of the correct VSS and suitable VSL. Hence, it is expected that the new algorithm would enhance traffic safety.

The continuity of the VSL system is important, as discontinuities in the system can aggravate traffic problems rather than improve flow. Figure 13 shows the variation in the selected VSS location among all stations on the road at 5-min intervals for both the JVSL and our EVSL algorithm, where the continuity of VSS is represented by a line graph. A station number of 0 means that the VSL is not operative. Figure 13a shows the results using JVSL, where many instances of zero values occurred during operation since the VSS was not selected within the required time and the algorithm was stopped due to the wrong algorithm of the VSS calculation. In contrast, Figure 13b shows the results of the EVSL algorithm, where good continuity was observed. Hence, this system is expected to provide stable VSL control in practical situations. 



Figure 13. Comparison of the VSL start station (VSS) continuity for the (a) existing JVSL and (b) EVSL algorithm. The $\mathrm{x}$ axis is the time ( $30 \mathrm{~s}$ interval). The $\mathrm{y}$ axis is the location of VSS. The number is the station number on the road. Zero (0) with dot line means there is no VSS station on the road.

\subsection{Speed Control Stability Assessment}

The wide distribution of speed can result in large variations in speed, which increases the risk of accidents on the freeway. The VSL system can keep the road safety by controlling the speed in the sections with the highest speed variations. To evaluate the safety of the proposed EVSL, we measured the speed variance on the tested freeway, as shown in Figures 14 and 15. To compare accurate situations applying the no VSL, JVSL, and EVSL, we experienced speed variance on the corresponding stations for the period from the beginning time to the ending time of the simulation.

As mentioned in Section 2.2, the JVSL has a problem to select the wrong congestion area. In addition, since the JVSL determines the distance to apply a VSL algorithm to freeway by the number of stations, instead of the station distance, it applies the VSL to longer sections of freeway than necessary. Hence, since the JVSL provides the wrong speed value at the wrong place as mentioned above, the speed variance of the JVSL is higher speed variance than that in the case of no VSL and EVSL being applied over the time period of 5:45 AM to 6:45 AM when the traffic congestion starts. The new EVSL algorithm showed slightly lower speed variance results than others over the same time period with proper congestion area selection and precise speed control. In the time period of 7:00 AM to 7:35 AM, the speed variance of the EVSL is higher than the JVSL because the EVSL has a traffic congestion later than the JVSL. 


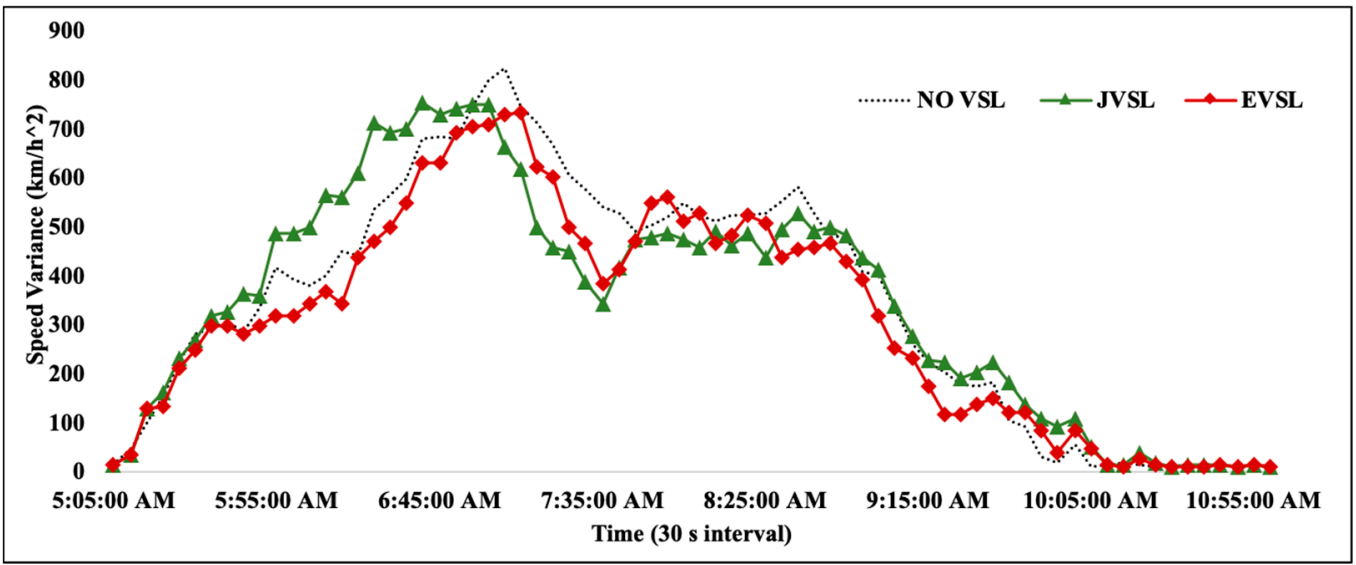

Figure 14. Speed variance over time. The $\mathrm{x}$ axis is the time ( $30 \mathrm{~s}$ interval). The $\mathrm{y}$ axis is the speed variance $\left(\mathrm{km} / \mathrm{h}^{2}\right)$.

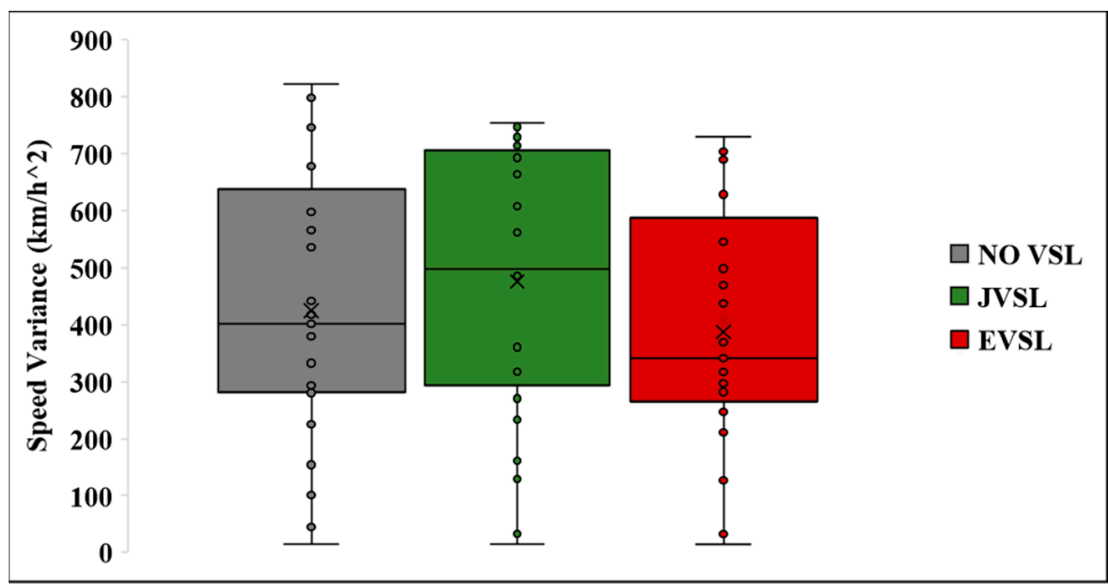

Figure 15. Variation analysis of total speed variance.

Figure 15 shows the comprehensive analysis of total speed variance over the time period of 5 AM to 11 AM. We can see that the JVSL shows a relatively high rate of speed variance in a wide distribution. On the other hand, since the EVSL shows a low rate of speed variance and narrower distribution than others, it proves that the EVSL performs more stable VSL calculations than others and controls traffic conditions more stably.

Figures 16 and 17 shows the average and max deceleration chart over the time period of 5 AM to 12 AM, which compares the existing JVSL, new EVSL, and no VSL. The deceleration, which is measured to evaluate the stability of traffic flow, is calculated by a difference between stations. We can expect that a high deceleration between stations means the vehicles are entering to the congestion area quickly and the drivers suddenly break a lot due to unstable traffic conditions. We prove the EVSL algorithm decrease the average deceleration of congestion area over the time period between 5:45 AM and 6:45 AM (the time when the congestion begins), as shown in Figures 16 and 18A. Especially, the EVSL algorithm shows the lower max deceleration than others from 5:45 AM to 8:00 AM, as shown in Figures 17 and 18B, because it sets the VSS to the correct location, which is the tail of congestion area and controls the vehicle speed through a correct calculation of the VSL speed. 


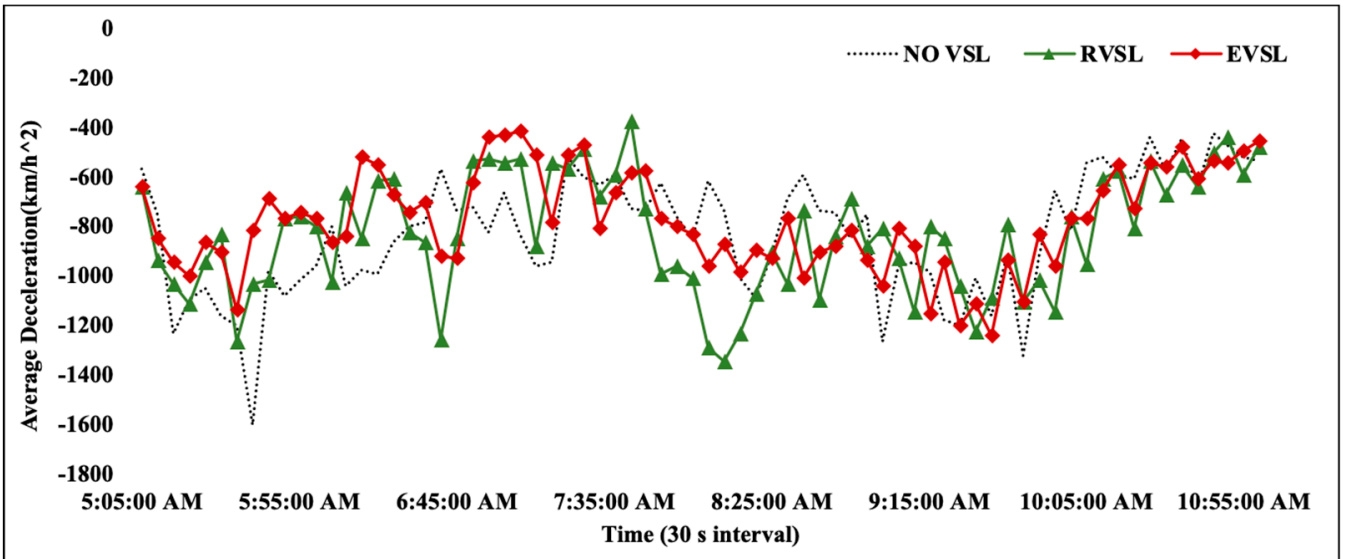

Figure 16. Average deceleration comparison between No variable speed limit (VSL), real-time VSL (RVSL), and enhanced VSL (EVSL). The $\mathrm{x}$-axis is the time (30 s intervals). The $\mathrm{y}$-axis is average deceleration $\left(\mathrm{km} / \mathrm{h}^{2}\right)$.

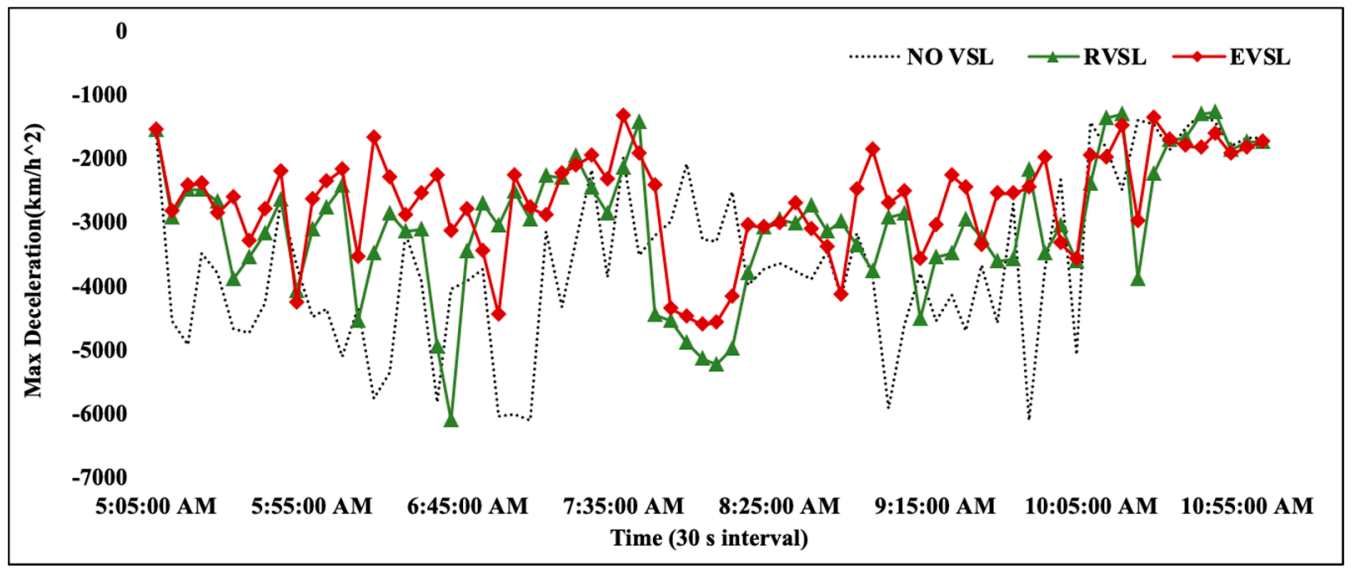

Figure 17. Max deceleration comparison between No VSL, RVSL, and EVSL. The x-axis is the time (30 s intervals). The $\mathrm{y}$-axis is max deceleration $\left(\mathrm{km} / \mathrm{h}^{2}\right)$.

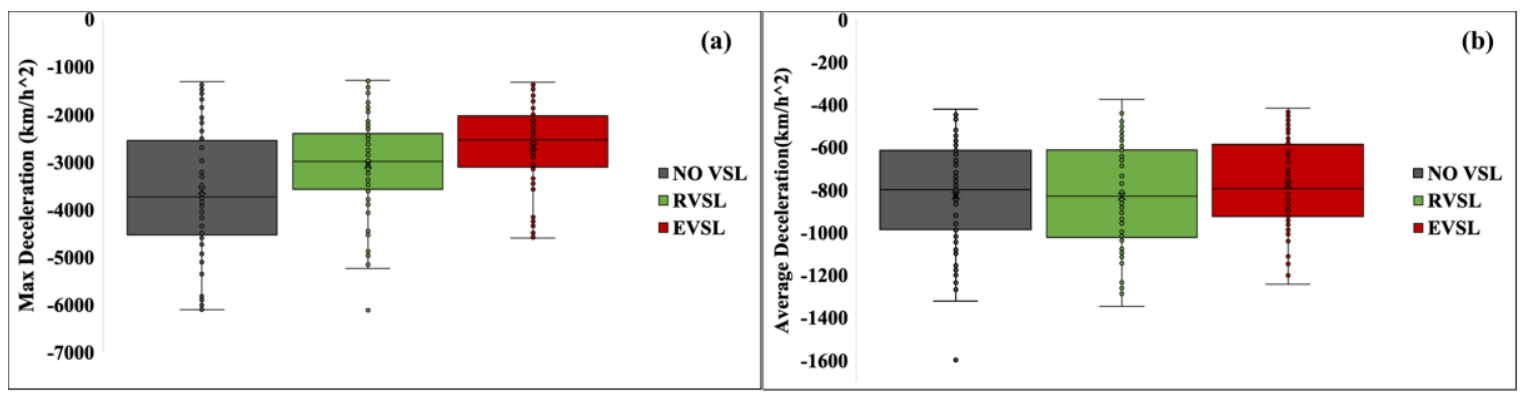

Figure 18. Max (a) and average (b) deceleration comparison between RVSL and EVSL.

\subsection{Total Travel Time and Shockwave}

Traffic control algorithms focus on the total travel time from the start point of the vehicle to the destination. The VSL system reduces the speed of vehicles before a congestion area in order to enhance road safety. However, if the VSL is applied over an excessively wide area, the real travel time could be increased unnecessarily without improving safety. The JVSL algorithm can significantly affect the total travel time because of the risk of operating irregular VSL due to a VSS decision error. Figures 19 and 20 compare the total travel times using the JVSL and the EVSL algorithm to the case of not using a VSL system. The JVSL resulted in a longer travel time (an increase of 1-3 min) compared to no VSL system. However, the EVSL algorithm showed a total travel time like no VSL. 


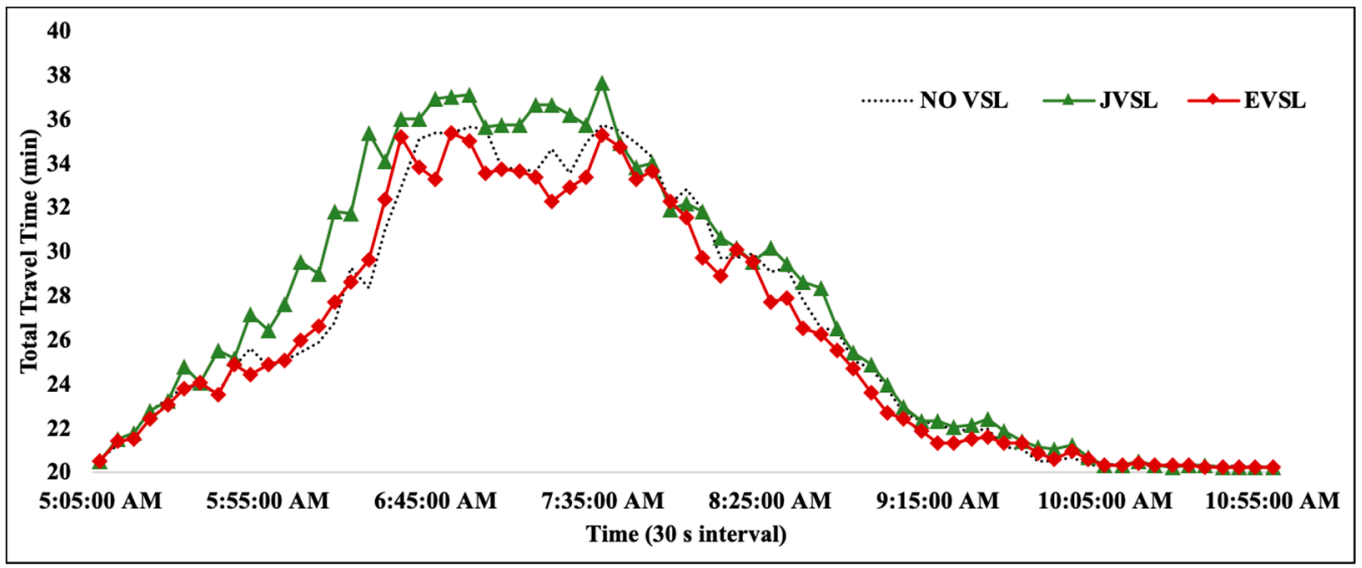

Figure 19. Total travel times for different traffic control algorithms. The x-axis is the time ( $30 \mathrm{~s}$ interval). The $y$-axis is the total travel time (min).

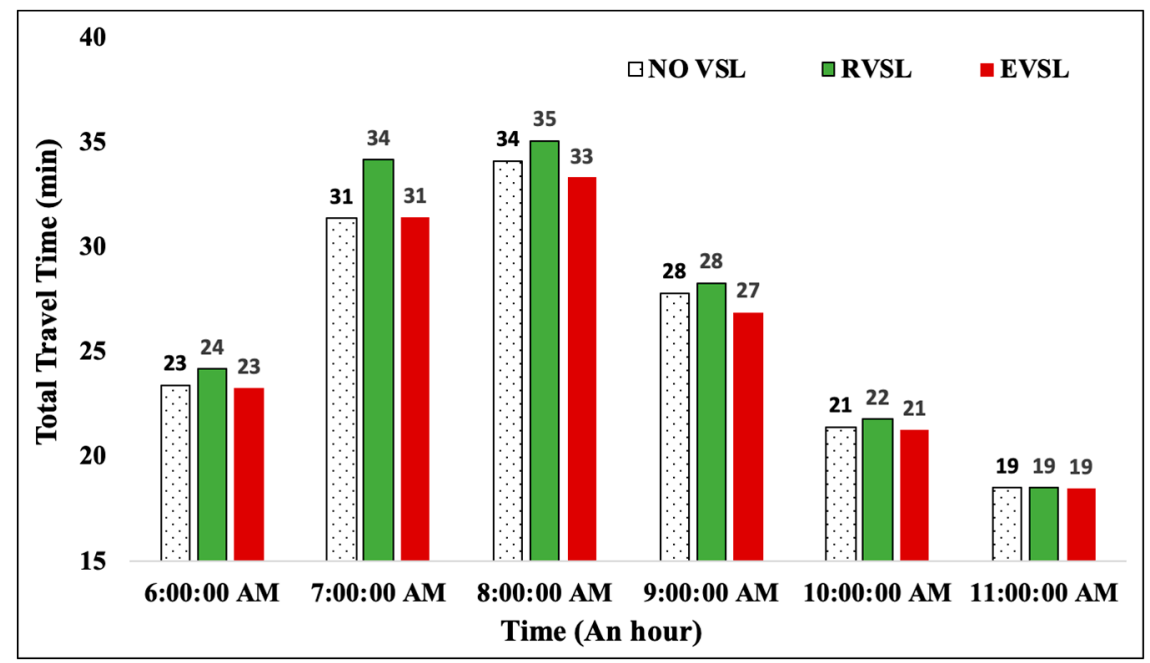

Figure 20. Total travel times for different traffic control algorithms by an hourly interval.

It has been proposed that the use of JVSL systems reduces the shockwave phenomenon in congestion areas. However, this conclusion was based on studies of short sections (1-2 km) of road. In the case of longer sections $(20-30 \mathrm{~km})$, there exists insufficient research since there are too many related variables and controlled sections to analyze. As shown in Figure 20, when compared to the non-VSL case, the new algorithm shows a reduced travel time (1-2 min for the peak time between 6:00 $\mathrm{AM}$ and 8:00 AM). This suggests that the shockwave phenomenon is alleviated. This phenomenon is continued up to the section of the traffic congestion. Since the new VSL algorithm operated stably with an accurate VSS, it is expected to reduce the risk of traffic accidents and the shockwave phenomenon.

\section{Conclusions and Future Work}

A new EVSL algorithm was proposed to overcome the limitations of the existing JVSL system and improve safety on freeways. The new algorithm accurately locates the VSS position, which enables it to correctly identify the sections of road to apply the required VSL. In addition, as based on the DMS distance, it effectively established the VSL range for traffic congested sections. Simulation results compared the JVSL system with the new algorithm. The existing algorithm showed many limitations due to its inaccurate selection of the VSS location, which resulted in aggravation of traffic congestion. In particular, analysis of speed control stability assessment and total travel time showed that the JVSL applied incorrect VSL operations in specific sections. Hence, the speed variance of the JVSL is higher speed variance for about $12 \%$ than that in the case of no VSL and EVSL being applied over the time 
period of 5:45 AM to 6:45 AM where the traffic congestion starts. A delay in the total travel time of an average of 1-2 min was observed comparing with no VSL. In contrast, the proposed EVSL algorithm produced an accurate VSS location and VSL value, decreasing the speed variance by $9 \%$ and the total travel time by 1-2 min when compared to the case of no VSL system. Hence, the proposed EVSL is expected to reduce the risk of accidents and alleviate shockwave phenomenon in the traffic congestion area. Lastly, reduced shockwaves also decreased the total travel time. Our algorithm can enhance freeway safety, along with traffic efficiency.

We also took advantage of the data provided by $\mathrm{Mn} / \mathrm{DoT}$ to obtain the threshold of the speed and acceleration for pVSS finding. They provide detailed distribution maps of speed and acceleration in the event of an incident. However, a threshold based on domestic data is needed to find the correct pVSS because the EVSL system is designed based on Korean freeways. Therefore, in a future work, we have a plan to collect incident data based on domestic data and the tail portion data of the congestion area based on the EVSL algorithm in operation in Korea. Based on the collected data, future studies will determine the appropriate threshold of speed and acceleration for domestic roads.

In addition, we will study a traffic control system where VSL is integrated with ramp metering where vehicles entering the freeway are controlled, as this is expected to provide a more effective traffic control solution.

Author Contributions: S.J. performed the research and wrote the paper. C.P. \& D.S. provided guidance for the research and revised the paper. All authors have read and agreed to the published version of the manuscript.

Funding: The study was funded by the Korea government (MSIT: Ministry of Science and ICT) (NRF-2020R1G1A1010166).

Acknowledgments: The National Research Foundation of Korea(NRF) grant supported this work.

Conflicts of Interest: The authors declare no conflict of interest.

\section{References}

1. Qureshi, K.N.; Abdullah, A.H. A survey on intelligent transportation systems. Middle-East J. Sci. Res. 2013, $15,629-642$.

2. Figueiredo, L.; Jesus, I.; Machado, J.T.; Ferreira, J.R.; De Carvalho, J.M. Towards the development of intelligent transportation systems. In Proceedings of the 2001 IEEE Intelligent Transportation Systems, Oakland, CA, USA, 25-29 August 2001; pp. 1206-1211.

3. Man, I.T.K.; Engineer, C.T. Intelligent Transport Systems. In Proceedings of the Better air Quality Motor Vehicle Control \& Technology Workshop, Hongkong, 18-20 September 2000.

4. Lee, C.; Hellinga, B.; Saccomanno, F. Evaluation of variable speed limits to improve traffic safety. Transp. Res. Part C Emerg. Technol. 2006, 14, 213-228. [CrossRef]

5. Allaby, P.; Hellinga, B.; Bullock, M. Variable speed limits: Safety and operational impacts of a candidate control strategy for freeway applications. EEE Trans. Intell. Transp. Syst. 2007, 8, 671-680. [CrossRef]

6. Rämä, P. Effects of weather-controlled variable speed limits and warning signs on driver behavior. Transp. Res. Rec. 1999, 1689, 53-59. [CrossRef]

7. Jo, Y.; Choi, H.; Jeon, S.; Jung, I. Variable speed limit to improve safety near traffic congestion on urban freeways. In Proceedings of the 2012 IEEE International Conference on Information Science and Technology, Wuhan, Hubei, China, 23-25 March 2012.

8. Wang, M.; Daamen, W.; Hoogendoorn, S.P.; van Arem, B. Connected variable speed limits control and car-following control with vehicle-infrastructure communication to resolve stop-and-go waves. J. Intell. Transp. Syst. 2016, 20, 559-572. [CrossRef]

9. Hegyi, A.; De Schutter, B.; Hellendoorn, H. Model predictive control for optimal coordination of ramp metering and variable speed limits. Transp. Res. Part C Emerg. Technol. 2005, 13, 185-209. [CrossRef]

10. Frejo, J.R.D.; Papamichail, I.; Papageorgiou, M.; De Schutter, B. Macroscopic modeling of variable speed limits on freeways. Transp. Res. Part C Emerg. Technol. 2019, 100, 15-33. [CrossRef]

11. Jeon, S.; Jung, I. Coordinated Ramp Metering for Minimum Waiting Time and Limited Ramp Storage. IEICE Trans. Fundam. Electron. Commun. Comput. Sci. 2016, 99, 1843-1855. [CrossRef] 
12. Immers, L.H.; Logghe, S. Traffic Flow Theory; Faculty of Engineering, Department of Civil Engineering, Section Traffic and Infrastructure: Kasteelpark, Arenberg, Germany, 2002.

13. Chen, C.; Petty, K.; Skabardonis, A.; Varaiya, P.; Jia, Z. Freeway performance measurement system: mining loop detector data. Transp. Res. Rec. 2001, 1748, 96-102. [CrossRef]

14. van Erp, P.B.; Knoop, V.L.; Hoogendoorn, S.P. Estimating the vehicle accumulation: Data-fusion of loop-detector flow and floating car speed data. In Proceedings of the 97th Transportation Research Board Annual Meeting, Washington, DC, USA, 7-11 January 2018.

15. Mathew, T.V.; Rao, K.V.K. Introduction to Transportation Engineering; Indian Institute of Technology: Bombay, India, 2006.

16. Kwon, E.; Park, C. Development of Freeway Operational Strategies with IRIS-in-Loop Simulation; Minnesota Department of Transportation: Saint Paul, MN, USA, 2012.

17. Korea Highway Corridor Information, Korea Expressway Corporation, Gimcheon. 2016. Available online: http://data.ex.co.kr (accessed on 24 March 2020).

18. PTV. VISSIM Version 5.2 Manual, Innovative Transportation Concepts; PTV Planung Transport Verkehr AG: Karlsruhe, Germany, 2009.

19. Han, Y.T.; Jeon, S.B.; Shin, S.J.; Seo, D.M.; Jung, I.B. Traffic Analysis and Simulation System for Korea Highway. KIISE Trans. Comput. Pract. 2016, 22, 426-440. [CrossRef]

20. Korea Highway Open Data, Korea Expressway Corporation. Available online: http://data.ex.co.kr (accessed on 24 March 2020).

21. Gomes, G.; May, A.; Horowitz, R. Congested freeway microsimulation model using VISSIM. Transp. Res. Rec. 2004, 1876, 71-81. [CrossRef]

22. Higgs, B.; Abbas, M.; Medina, A. Analysis of the Wiedemann car following model over different speeds using naturalistic data. In Proceedings of the Procedia of RSS Conference, Indianapolis, IN, USA, 1-22 September 2011.

23. Fellendorf, M.; Vortisch, P. Validation of the microscopic traffic flow model VISSIM in different real-world situations. In Proceedings of the 80th Annual Meeting of the Transportation Research Board, Washington, DC, USA, 7-11 January 2001.

24. Shin, S.J.; Lee, C.S.; Han, Y.T.; Jeon, S.B.; Seo, D.M.; Jung, I.B. Calibration for Simulating a ITS Algorithm in Korea Highway. In Proceedings of the Korea Information Processing Society Conference, Busan, Korea, 4-5 November 2016; pp. 443-446.

(C) 2020 by the authors. Licensee MDPI, Basel, Switzerland. This article is an open access article distributed under the terms and conditions of the Creative Commons Attribution (CC BY) license (http://creativecommons.org/licenses/by/4.0/). 